\title{
Photonuclear reactions in astrophysical p-process: Theoretical calculations and experiment simulation based on ELI-NP
}

\author{
$\mathrm{Yi} \mathrm{Xu}^{1, \mathrm{a}}$, Wen $\mathrm{Luo}^{2}$, Dimiter Balabanski ${ }^{1}$, Stephane Goriely ${ }^{3}$, Catalin Matei $^{1}$, and Ovidiu Tesileanu ${ }^{1}$ \\ 1 Extreme Light Infrastructure-Nuclear Physics, 077125 Magurele, Ilfov, Romania \\ 2 School of Nuclear Science and Technology, University of South China, 421001 Hengyang, China \\ 3 Institut d'Astronomie et d'Astrophysique, CP. 226, Universite Libre de Bruxelles, 1050 Brussels, Belgium
}

\begin{abstract}
The astrophysical p-process is an important way of nucleosynthesis to produce the stable and proton-rich nuclei beyond $\mathrm{Fe}$ which can not be reached by the s- and r-processes. In the present study, the astrophysical reaction rates of $(\gamma, n),(\gamma, p)$, and $(\gamma, \alpha)$ reactions are computed within the modern reaction code TALYS for about 3000 stable and proton-rich nuclei with $12<\mathrm{Z}<110$. The nuclear structure ingredients involved in the calculation are determined from experimental data whenever available and, if not, from global microscopic nuclear models. In particular, both of the Wood-Saxon potential and the double folding potential with density dependent M3Y (DDM3Y) effective interaction are used for the calculations. It is found that the photonuclear reaction rates are very sensitive to the nuclear potential, and the better determination of nuclear potential would be important to reduce the uncertainties of reaction rates. Meanwhile, the Extreme Light Infrastructure-Nuclear Physics (ELI-NP) facility is being developed, which will provide the great opportunity to experimentally study the photonuclear reactions in p-process. Simulations of the experimental setup for the measurements of the photonuclear reactions ${ }^{96} \mathrm{Ru}(\gamma, \mathrm{p})$ and ${ }^{96} \mathrm{Ru}(\gamma, \alpha)$ are performed. It is shown that the experiments of photonuclear reactions in p-process based on ELI-NP are quite promising.
\end{abstract}

\section{Introduction}

In massive star evolution and stellar explosive site, the astrophysical p-process is an important way of nucleosynthesis to produce the stable and proton-rich nuclei beyond $\mathrm{Fe}$ which cannot be reached by the $\mathrm{s}$ - and r-processes. The common picture is that the p-nuclei are synthesized by photodisintegration of pre-existing s- and r-processes nuclei via the $(\gamma, n),(\gamma, p)$, and $(\gamma, \alpha)$ reaction channels. The dominant nuclear flows of p-process go towards the neutron-deficient region through $(\gamma, n)$ reactions. Along the isotopic paths, the proton separation energies become progressively smaller, while the corresponding energies for the neutrons go up. As a result, the flows to more neutron-deficient isotopes are hindered, and sometimes are deflected by $(\gamma, \mathrm{p})$, and $(\gamma, \alpha)$ reactions [1]. The typical temperature of p-process is between $\mathrm{T}_{9}=1.5$ and $\mathrm{T}_{9}=3.5$.

To completely determine the nucleosynthesis of p-process, accurate knowledge of capture and photodisintegration reaction rates for about 3000 stable and protonrich nuclei is essential. However, available experimental information for the reactions involved in p-process is quite limited [2]. For the reactions whose experimental data are not available yet, the state-of-the-art nuclear reaction models and the nuclear structure knowledge deduced by microscopic models should be taken into account for the evaluations of reaction rates.

In the present study, we perform the systematic computations of $(\gamma, n),(\gamma, p)$, and $(\gamma, \alpha)$ reaction rates on

a e-mail: yi.xu@eli-np.ro about 3000 nuclei based on the TALYS software with the specific nuclear structure ingredients. Furthermore, simulations of the experimental setup for the measurements of photonuclear reactions of astrophysics interest in p-process based on the Extreme Light Infrastructure-Nuclear Physics (ELI-NP) facility are performed, and the preliminarily results are correspondingly presented.

\section{Systematic calculations of photonuclear reaction rates}

The photonuclear reaction rates of $(\gamma, \mathrm{n}),(\gamma, \mathrm{p})$, and $(\gamma, \alpha)$ on about 3000 stable and proton-rich nuclei are computed with the help of TALYS, which is a software for the simulation of nuclear reactions $[3,4]$. TALYS provides a complete description of all reaction channels and observables and, in particular, takes into account all types of direct, pre-equilibrium, and compound mechanisms to estimate the total reaction probability as well as the competition between the various open channels. For the nuclear ingredients used in the TALYS calculation, the nuclear inputs required for calculations can be extracted from basic nuclear-structure properties. Whenever available, all experimental information on nuclear masses, deformation, and spectra of low-lying states is considered, in particular the RIPL-3 database [5]. If not, local and global inputs derived by various nuclearstructure models have been taken into account. For a reliable prediction, such models need to be as microscopic as possible, especially when dealing with the exotic proton-rich nuclei for which extrapolation is required. Such a microscopic description provided by physically

(C) The Authors, published by EDP Sciences. This is an Open Access article distributed under the terms of the Creative Commons Attribution License 4.0 (http://creativecommons.org/licenses/by/4.0/). 


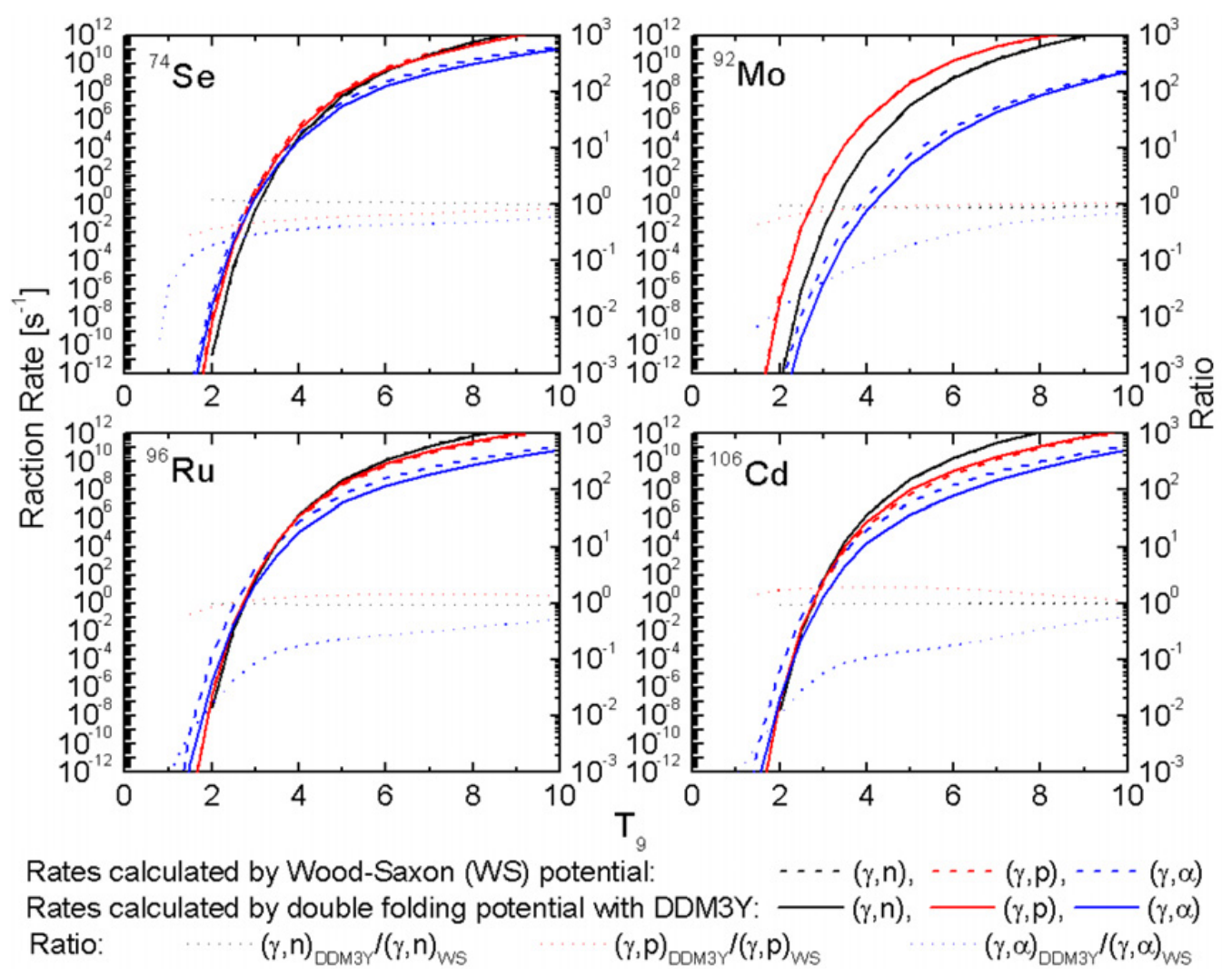

Figure 1. Photonuclear reaction rates on the targets of ${ }^{74} \mathrm{Se},{ }^{92} \mathrm{Mo},{ }^{96} \mathrm{Ru}$ and ${ }^{106} \mathrm{Cd}$ calculated by using Wood-Saxon (WS) potential and double folding potential with density dependent M3Y (DDM3Y) effective interaction, as well as the ratios of the 2 sets of reaction rates shown as a function of $\mathrm{T}_{9}$.

sound theories based on first principles likely renders extrapolations away from experimentally known energy or mass regions more reliable than predictions derived from more-or-less parametric phenomenological approaches of various types and levels of sophistication.

In the present calculations, the nuclear masses are taken from the 2012 Atomic Mass Evaluation [6] and Hartree-Fock-Bogoliubov (HFB) method based on the BSk21 Skyrme interaction [7]. The $\gamma$-ray strength functions obtained from the HFB + QRPA models based on the BSk7 Skyrme force are considered [8]. The nuclear level densities derived by the microscopic HFB plus a combinatorial approach are taken into account [9]. For comparison, both of the Wood-Saxon (WS) potential $[10,11]$ and double folding potential with density dependent M3Y (DDM3Y) effective interaction [12] are used in the calculations. Figure 1 shows the photonuclear reaction rates on the targets of ${ }^{74} \mathrm{Se},{ }^{92} \mathrm{Mo},{ }^{96} \mathrm{Ru}$ and ${ }^{106} \mathrm{Cd}$ calculated by the two types of potentials and the ratios of the reaction rates as a function of $\mathrm{T}_{9}$. We can see that, at the typical temperature range of p-process from $\mathrm{T}_{9}=1.5$ to $\mathrm{T}_{9}=3.5$, the ratios of $(\gamma, \alpha)$ reaction rates calculated by WS potential to the rates calculated by double folding potential with DDM3Y interaction decrease by the factor of 10 to 100 for the four targets nuclei.

Furthermore, the $(\gamma, n),(\gamma, p)$ and $(\gamma, \alpha)$ reaction rates at $\mathrm{T}_{9}=2.5$ calculated by the double folding potential with DDM3Y interaction for about 3000 stable and protonrich nuclei are shown on the $(\mathrm{N}, \mathrm{Z})$ plane in Fig. 2 . We also compare the $(\gamma, \alpha)$ reaction rates by showing the ratio of the reaction rates calculated by WS potential and double folding potential with DDM3Y interaction in
Fig. 2, and it is found that the $(\gamma, \alpha)$ reaction rates are very sensitive to the nuclear potential. Therefore, we propose to experimentally study the photonuclear reactions based on the ELI-NP facility, especially for the $(\gamma, \alpha)$ channel. The nuclear potential can be extracted by fitting the measured photonuclear cross sections, which could be used to better determine the $(\gamma, \alpha)$ reaction rates.

\section{Simulations of the experimental setup for the measurements of photonuclear reactions based on ELI-NP facility}

The Extreme Light Infrastructure-Nuclear Physics (ELI$\mathrm{NP}$ ) is aiming to use extreme electromagnetic fields for nuclear physics research. The facility will comprise a high power laser system and a very brilliant gamma beam system. The technology involved in the construction of both systems is at the limits of the present-day's technological capabilities. The gamma beam, produced via Compton backscattering of a laser beam on a relativistic electron beam, will be characterized by a narrow bandwidth $(<0.5 \% \mathrm{bdw})$ and tunable energy of up to almost $20 \mathrm{MeV}$. The brilliance of the gamma beam at the peak energy could reach $10^{23} / \mathrm{s} \mathrm{mm}^{2} \mathrm{mrad}^{2}$ with $0.1 \%$ bdw. The main features of the gamma beams to be delivered at ELI-NP can be found in Ref. [13]. The research program of the facility covers a broad range of key topics in frontier fundamental physics and new nuclear physics. In particular, the research of nuclear astrophysics based on gamma-induced reactions with very low cross sections will largely benefit form the use of the high intensity gamma beams at ELI-NP. 


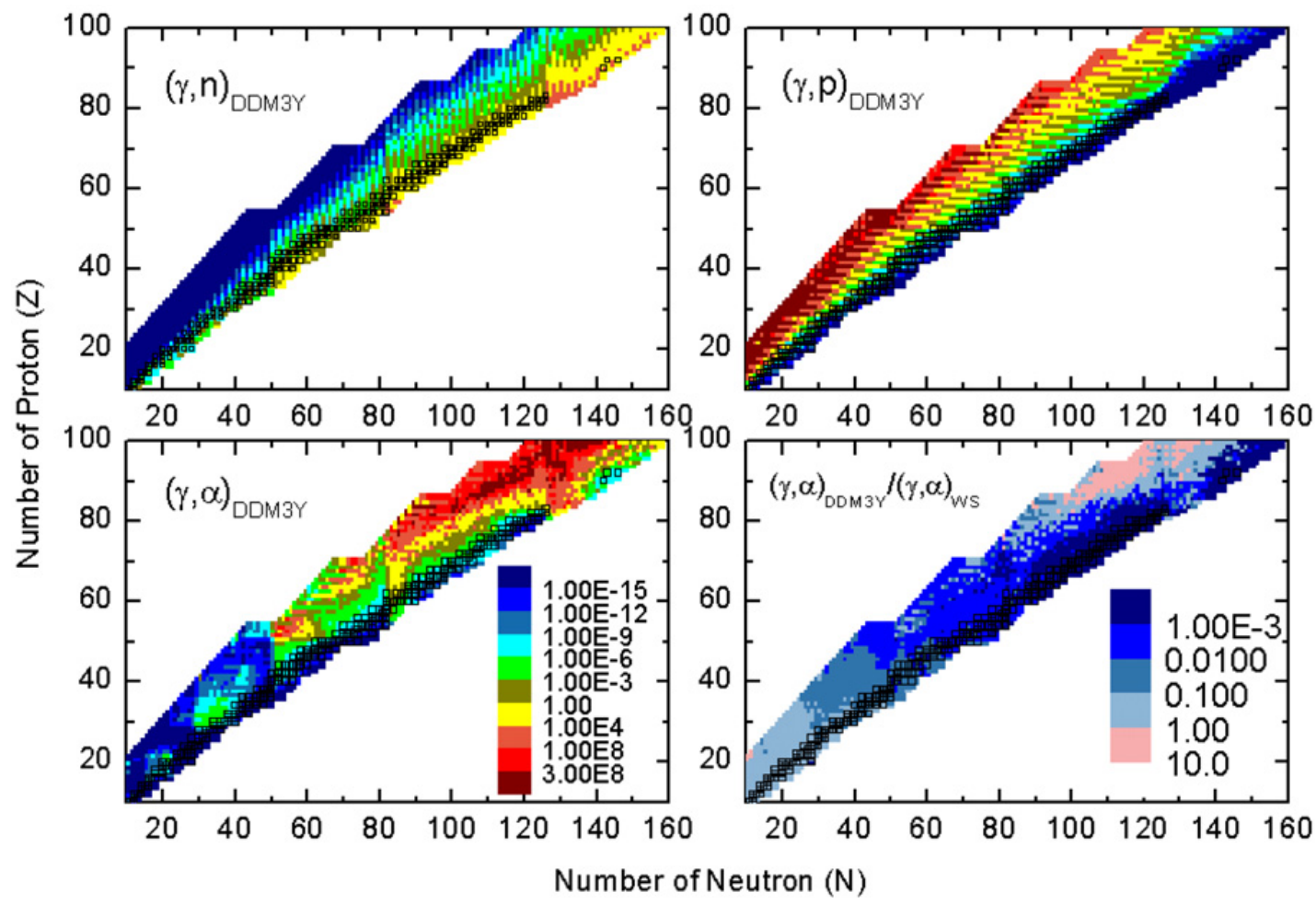

Figure 2. Representation in the (N,Z) plane of the $(\gamma, \mathrm{n}),(\gamma, \mathrm{p})$, and $(\gamma, \alpha)$ reaction rates calculated by double folding potential with DDM3Y interaction at $\mathrm{T}_{9}=2.5$; and the ratios of $(\gamma, \alpha)$ rates calculated by double folding potential with DDM3Y interaction to those calculated by Wood-Saxon potential at $\mathrm{T}_{9}=2.5$ for all the 3000 stable and proton-rich nuclei.
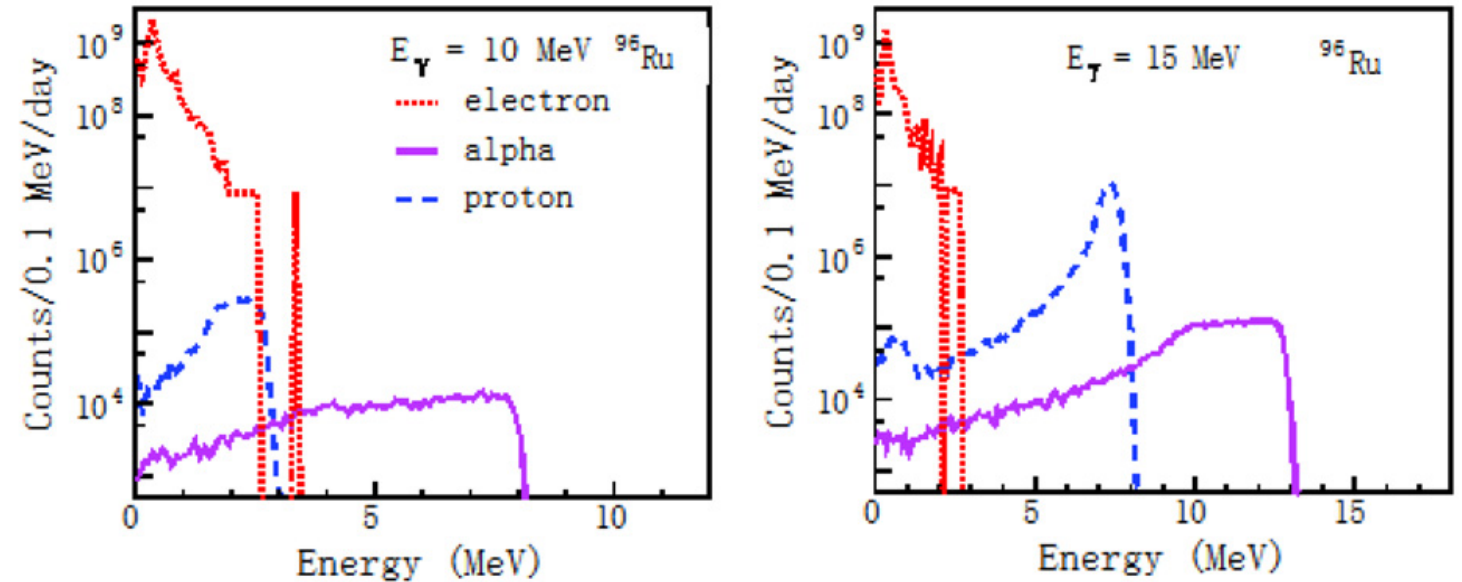

Figure 3. Energy spectrum of proton and alpha particles for ${ }^{96} \mathrm{Ru}(\gamma, \mathrm{p})$ and ${ }^{96} \mathrm{Ru}(\gamma, \alpha)$ reactions obtained by GEANT4 simulations. The incident gamma-ray energies of $10 \mathrm{MeV}$ and $15 \mathrm{MeV}$ are used. The target thickness of Ruthenium is $10 \mu \mathrm{m}$.

Meanwhile, for the detection system of charged particles, silicon detectors represent one of the best solution for their detection because they can guarantee the exceptional energy resolution and they have essentially $100 \%$ efficiency in charged particle detection. In the case of photonuclear reactions of astrophysical relevance, the emitted charged-particles have low energies, ranging from few hundreds $\mathrm{keV}$ to few $\mathrm{MeV}$. Therefore, lowthreshold detectors are necessary and particle identification techniques have presently proven impossible, which makes kinematical identification the only viable option to separate the reaction of interest from others.

A silicon strip detector array, named ELISSA, will be realized in a common effort by ELI-NP and INFN Laboratori Nazionali del Sud (INFN-LNS) in Catania, Italy. The prototype of ELISSA was built and tested at
INFN-LNS [14]. On this occasion, the simulations of the experimental setup for the measurements of $(\gamma, \alpha)$ and $(\gamma, \mathrm{p})$ reactions on ${ }^{96} \mathrm{Ru}$ target are performed with the help of GEANT4. In the simulation, the configuration of ELISSA is taken into account, and the thickness of ${ }^{96} \mathrm{Ru}$ target is $10 \mu \mathrm{m}$ [15]. For the incident gamma-ray energies of $10 \mathrm{MeV}$ and $15 \mathrm{MeV}$, the energy spectrum of proton and $\alpha$-particle for ${ }^{96} \mathrm{Ru}(\gamma, \mathrm{p})$ and ${ }^{96} \mathrm{Ru}(\gamma, \alpha)$ reactions are shown in Fig. 3. One can find that $10^{4}$ counts per day can be obtained at $\mathrm{E}_{\gamma}=10 \mathrm{MeV}$ for the experimental yield of $\alpha$-particle, and $10^{5}$ counts per day can be obtained at $\mathrm{E}_{\gamma}=15 \mathrm{MeV}$. We believe that the experiment of the simulated photonuclear reaction is quite promising, and it would be helpful to constraint the $\alpha$-particle potential used for the determination of astrophysical reaction rates. 


\section{References}

[1] M. Arnould and S. Goriely, Phys. Rep. 384, 1 (2003)

[2] T. Szücs et al., Nuclear Data Sheets 120, 191 (2014)

[3] A.J. Koning, S. Hilaire, M.C. Duijvestijn, NRGreport 21297/04.62741/P (2004)

[4] Y. Xu, S. Goriely, A.J. Koning, S. Hilaire, Phys. Rev. C 90, 024604 (2014)

[5] R. Capote et al., Nuclear Data Sheets 110, 3107 (2009)

[6] G. Audi et al., Chin. Phys. C 361287 (2012)

[7] S. Goriely, N. Chamel, J.M. Pearson, Phys. Rev. C 82, 035804 (2010)
[8] S. Goriely, E. Khan, M. Samyn, Nucl. Phys. A 739, 331 (2004)

[9] S. Goriely, S. Hilaire, A.J. Koning, Phys. Rev. C 78, 064307 (2008)

[10] S. Watanabe, Nucl. Phys. 8, 484 (1958)

[11] A.J. Koning, J.P. Delaroche, Nucl. Phys. A 713, 231 (2003)

[12] P. Demetriou, C. Grama, S. Goriely, Nucl. Phys. A 707, 253 (2002)

[13] C.A. Ur et al., Nucl. Inst. Meth. B 355, 198 (2015)

[14] M. La Cognata et al., accepted by Journal of Instrumentation (2016)

[15] W. Luo, H.Y. Lan, Y. Xu et al., Nucl. Inst. Meth. A 849, 49 (2017) 